\title{
Biofortification: how can we exploit plant science and biotechnology to reduce micronutrient deficiencies?
}

\author{
Irene Murgia ${ }^{1 *}$, Laura De Gara ${ }^{2 *}$ and Michael A. Grusak ${ }^{3 *}$ \\ ${ }^{1}$ Department of Biosciences, Università degli Studi di Milano, Milano, Italy \\ ${ }^{2}$ Centro Integrato di Ricerca, Università Campus Bio-Medico di Roma, Roma, Italy \\ ${ }^{3}$ Department of Pediatrics, USDA-ARS Children's Nutrition Research Center, Baylor College of Medicine, Houston, TX, USA \\ *Correspondence: irene.murgia@unimi.it; L.Degara@unicampus.it; mgrusak@bcm.edu
}

Edited by:

Steven C. Huber, USDA-ARS, USA

Keywords: biofortification, human nutrition, micronutrients, mineral deficiencies, vitamin deficiencies

Throughout the developing world, the long-term consequences of insufficient amounts of essential micronutrients in the human diet can be more devastating than low energy intake. Micronutrients are involved in all aspects of development, growth, and physiology of the human body (including from early embryonic stage), and their deficiencies can cause birth defects, permanent physical and mental impairment, as well as an increased risk of death by infectious and chronic diseases. As plant scientists, we are now facing challenging and urgent goals: how to feed the world's increasing population and how to feed it better. In other words, we need to produce more plant food in sustainable ways and such food should be of the highest nutritional value.

This e-Book aims to provide the most recent advances on plant biofortification for micronutrients as well as a comprehensive overview of the different approaches that can be pursued for producing micronutrient-rich staple plants. We list here some of the major points arising from these papers.

\section{VITAMINS}

Vitamins are organic compounds that are required in limited amounts for normal human growth and activity and that cannot be synthesized by the human body. Various genetic engineering approaches for increasing the concentrations of vitamin $B_{1}, B_{6}$, $\mathrm{C}$, or D in edible parts of the plant are discussed, together with the possible effects of such increases on plant tolerance to stress.

Acute deficiency in Vitamin $B_{1}$ (thiamin) can result in fatal neurological and cardiovascular disorders. Pourcel et al. (2013) highlight the fact that although the main source of vitamin $B_{1}$ in the diet is plants, there is still a need for enhancing thiamin levels in crop plants because a human diet based mainly on the edible portions of the staple crops, rice, wheat, or maize, is poor in vitamin $B_{1}$. In addition, the refining process for producing white flour and polished rice further reduces their final vitamin $B_{1}$ content. The thiamin biosynthetic pathway is quite complex in plants, utilizing enzymes regulated in a highly complicated fashion, and involving RNA sequences called riboswitches. The most updated pathway scheme achieved in the model plant Arabidopsis thaliana is reported in Pourcel et al. (2013), where the various biofortification approaches are discussed in light of the complexity of the pathway itself.

Vitamin $\mathrm{B}_{6}$ is involved in different biochemical pathways and in diverse physiological roles in humans that are related to hormones, the immune system, and vascular functions. Adequate vitamin $B_{6}$ intake is beneficial for humans since it reduces the incidence of various health threats, including several multifactorial neurological disorders (Vanderschuren et al., 2013). Differently from vitamin $\mathrm{B}_{1}$, de novo plant vitamin $\mathrm{B}_{6}$ biosynthesis involves only two enzymes, PDX1 and PDX2 (pyridoxin synthesis gene 1 and 2). Vanderschuren et al. (2013) comment on the first successful results in engineering vitamin $B_{6}$ biofortification by overexpression of both enzymes in Arabidopsis. These authors suggest that next steps will be the full understanding of the regulation of vitamin $\mathrm{B}_{6}$ biosynthesis, the selection of target food crops, and use of biofortification strategies (including the one applied in Arabidopsis) to such food crops.

Vitamin D maintains and regulates calcium levels in the body. Vitamin $\mathrm{D}_{3}$ biosynthesis requires UV-B rays for its photochemical conversion from pro-vitamin $\mathrm{D}_{3}$, a process occurring in the human body; therefore, depending on latitude or season, people may be at risk for vitamin D deficiency if sufficient vitamin $\mathrm{D}$ is not consumed through supplementation or through a vitamin D-rich diet. Fish are good sources of vitamin D, whereas only small amounts of vitamin D can be found in plants (Jäpelt and Jakobsen, 2013). Nonetheless, Jäpelt and Jakobsen (2013) argue that increased knowledge of vitamin D and of its metabolites in plants, as well as improvements in analytical methods for detection of vitamin $\mathrm{D}$ and of its derivatives, can offer new tools for increasing vitamin D content in edible parts of plants. Furthermore, a hypothetical biosynthetic pathway for vitamin D in plants is presented by these authors.

Plant foods are the main dietary source of vitamin C (ascorbic acid; ASC) for humans, for whom severe deficiency causes scurvy and can lead to death. Sub-optimal vitamin C intake, by leading to increased susceptibility to infections and diseases, can represent a health threat for both developing and developed world populations. In the review by Locato et al. (2013), possible plant biofortification strategies for this vitamin are examined, while acknowledging that ASC levels in plants should be considered a complex quantitative trait. Interestingly, ASC levels are tightly linked not only to plant tolerance to stress, but also to sugar biosynthesis and to the regulation of the fluxes of ASC precursors among metabolic pathways (Locato et al., 2013). Transgenic tomato plants overepressing a bacterial pyrophosphatase show increased expression of some genes coding for enzymes involved in the major ASC biosynthetic route in plants (Smirnoff-Wheeler pathway) and such transgenic plants show increased ASC content 
in fruits, as well as an increase of major sugars (sucrose and glucose) and a decrease in starch content (Osorio et al., 2013).

\section{METAL MICRONUTRIENTS}

Iron $(\mathrm{Fe})$ and zinc $(\mathrm{Zn})$ are two essential metal micronutrients for human health; their deficiencies in the human diet contribute to high rates of mortality in developing countries. Manganese (Mn) deficiency, though less prevalent than $\mathrm{Fe}$ and $\mathrm{Zn}$ deficiency, can also lead to serious health problems, including birth defects (Bashir et al., 2013). A review of progress in biofortification of rice with such micronutrients is presented in Bashir et al. (2013), where a list of possible bottlenecks for biofortification are also presented, including the avoidance of accumulation of the toxic metal cadmium. The need for better knowledge of seed physiology and morphology is also discussed, in order to ensure successful increases in micronutrient concentrations in the edible parts of plant seeds, especially after the polishing and refining processes are completed. Along these lines, Blair et al. (2013) studied seed coats of common bean seeds to characterize variations in $\mathrm{Fe}$ and $\mathrm{Zn}$ concentrations in individual lines of a recombinant inbred population. They use these results to identify some of the underlying genetic loci responsible for seed coat accumulation of these metals, which should be useful for future biofortification efforts with bean.

Success in increasing $\mathrm{Fe}$ and $\mathrm{Zn}$ concentrations in polished seeds of a major rice variety in Myanmar, through a transgenic approach targeting metal transport and accumulation, is presented by Aung et al. (2013). In another study, increases in Fe concentration in a Japonica rice variety were also achieved by stacking a set of Fe homeostasis genes (Masuda et al., 2013). Because several iron-related processes were altered (from uptake to chelation and storage), the authors were careful to elevate seed Fe levels without inducing symptoms of Fe deficiency at the whole-plant level. Moreover, they used a marker-free vector that could serve to increase public acceptance of the transformed lines (Masuda et al., 2013). This so-called "push-pull" mechanism of augmenting Fe uptake at one end of the system, along with the storage sink for Fe at the other end of the system (by means of overexpressing Fe transport and storage genes), was investigated in detail in a different transgenic rice line and demonstrated not to interfere with general Fe homeostasis in such plants, relative to non-transgenic controls (Wang et al., 2013). Similar nontarget effects of metal-related genes were studied in maize plants expressing a soybean ferritin gene (iron storage protein) in the endosperm (Kanobe et al., 2013). These authors demonstrate that the levels of some metal-related gene transcripts and proteins were indeed changed in the transgenic line, emphasizing the need to take a holistic approach when evaluating transgenic events.

Three different gene families have been described in detail and proposed as possible targets for future $\mathrm{Zn}$ or Fe biofortification strategies: the NAC transcription factors (Ricachenevsky et al., 2013a), the metal tolerance proteins (MTP's) (Ricachenevsky et al., 2013b) and the ZIP family of metal transporters (Astudillo et al., 2013). Notably, a candidate gene for increasing $\mathrm{Zn}$ concentration in common bean, PvZIP12, is proposed in Astudillo et al. (2013) and a model has been proposed for the role of the rice OsNAC5 gene in senescence and metal re-mobilization (Ricachenevsky et al., 2013a).

\section{IODINE}

Iodine is a non-metal micronutrient that is essential for human health and whose deficiency impairs thyroid functions. When severe deficiency occurs, fetal development can be affected with consequent irreversible brain damage and mental retardation. A possible approach to produce plants biofortified with iodine is to administer exogenous iodine salts to the soil during plant growth. Kato et al. (2013) demonstrate that rice roots possess the ability to reduce $\mathrm{IO}_{3}^{-}$to $\mathrm{I}^{-}$and that such reduction is dependent, at least in part, on external iodine conditions. Such results suggest the existence of an iodate reductase in plant roots (Kato et al., 2013). Moreover, Kiferle et al. (2013) demonstrate that tomato fruits can be good targets for iodine fortification, by means of plant irrigation with $\mathrm{KIO}_{3}$ during growth.

\section{MOVING FORWARD}

The papers presented in this e-Book demonstrate how knowledge in plant metabolism, physiology, and molecular biology can provide approaches for increasing the nutritional value of plant derived foods. The contributions also draw attention to the need for multidisciplinary efforts to cope with the challenges of food security and micronutrient malnutrition. We believe that the information presented in this e-Book will provide several novel ideas and will stimulate new directions amongst researchers in the field of plant biofortification. We also hope that these contributions can serve as a good source of background knowledge to educate and bring new scientists into the field. We are indebted to the many authors who have contributed to this e-Book and for their continued work in this important field of science. We feel privileged to have had the opportunity to oversee and edit this fine group of papers.

\section{REFERENCES}

Astudillo, C., Fernandez, A. C., Blair, M. W., and Cichy, K. A. (2013). The Phaseolus vulgaris ZIP gene family: identification, characterization, mapping, and gene expression. Front. Plant Sci. 4:286. doi: 10.3389/fpls.2013.00286

Aung, M. S., Masuda, H., Kobayashi, T., Nakanishi, H., Yamakawa, T., and Nishizawa, N. K. (2013). Iron biofortification of Myanmar rice. Front. Plant Sci. 4:158. doi: 10.3389/fpls.2013.00158

Bashir, K., Takahashi, R., Nakanishi, H., and Nishizawa, N. K. (2013). The road to micronutrient biofortification of rice: progress and prospects. Front. Plant Sci. 4:15. doi: 10.3389/fpls.2013.00015

Blair, M. W., Izquierdo, P., Astudillo, C., and Grusak, M. A. (2013). A legume biofortification quandary: variability and genetic control of seed coat micronutrient accumulation in common beans. Front. Plant Sci. 4:275. doi: 10.3389/fpls.2013.00275

Jäpelt, R. B., and Jakobsen, J. (2013). Vitamin D in plants: a review of occurrence, analysis, and biosynthesis. Front. Plant Sci. 4:136. doi: 10.3389/fpls. 2013.00136

Kanobe, M. N., Rodermel, S. R., Bailey, T., and Scott, M. P. (2013). Changes in endogenous gene transcript and protein levels in maize plants expressing the soybean ferritin transgene. Front. Plant Sci. 4:196. doi: 10.3389/fpls.2013.00196

Kato, S., Wachi, T., Yoshihira, K., Nakagawa, T., Ishikawa, A., Takagi, D., et al. (2013). Rice (Oryza sativa L.) roots have iodate reduction activity in response to iodine. Front. Plant Sci. 4:227. doi: 10.3389/fpls. 2013.00227

Kiferle, C., Gonzali, S., Holwerda, H. T., Ibaceta, R. R., and Perata, P. (2013). Tomato fruits: a good target for iodine biofortification. Front. Plant Sci. 4:205 doi: 10.3389/fpls.2013.00205 
Locato, V., Cimini, S., and De Gara, L. (2013). Strategies to increase vitamin C in plants: from plant defense perspective to food biofortification. Front. Plant Sci. 4:152. doi: 10.3389/fpls.2013.00152

Masuda, H., Kobayashi, T., Ishimaru, Y., Takahashi, M., Aung, M. S., Nakanishi, H., et al. (2013). Iron-biofortification in rice by the introduction of three barley genes participated in mugineic acid biosynthesis with soybean ferritin gene. Front. Plant Sci. 4:132. doi: 10.3389/fpls.2013.00132

Osorio, S., Nunes-Nesi, A., Stratmann, M., and Fernie, A. R. (2013). Pyrophosphate levels strongly influence ascorbate and starch content in tomato fruit. Front. Plant Sci. 4:308. doi: 10.3389/fpls.2013.00308

Pourcel, L., Moulin, M., and Fitzpatrick, T. B. (2013). Examining strategies to facilitate vitamin $\mathrm{B}_{1}$ biofortification of plants by genetic engineering. Front. Plant Sci. 4:160. doi: 10.3389/ fpls.2013.00160

Ricachenevsky, F. K., Menguer, P. K., and Sperotto, R. A. (2013a). kNACking on heaven's door: how important are NAC transcription factors for leaf senescence and Fe/Zn remobilization to seeds. Front. Plant Sci. 4:226. doi: 10.3389/ fpls.2013.00226

Ricachenevsky, F. K., Menguer, P. K., Sperotto, R. A., Williams, L. E., and Fett, J. P. (2013b). Roles of plant metal tolerance proteins (MTP) in metal storage and potential use in biofortification strategies. Front. Plant Sci. 4:144. doi: 10.3389/fpls.2013.00144
Vanderschuren, H., Boycheva, S., Li, K.-T., Szydlowski, N., Gruissem, W., and Fitzpatrick, T. B. (2013). Strategies for vitamin B6 biofortification of plants: a dual role as a micronutrient and a stress protectant. Front. Plant Sci. 4:143. doi: 10.3389/fpls.2013.00143

Wang, M., Gruissem, W., and Bhullar, N. K. (2013). Nicotianamine synthase overexpression positively modulates iron homeostasis-related genes in high iron rice. Front. Plant Sci. 4:156. doi: 10.3389/fpls.2013.00156

Received: 02 October 2013; accepted: 10 October 2013; published online: 06 November 2013.

Citation: Murgia I, De Gara L and Grusak MA (2013) Biofortification: how can we exploit plant science and biotechnology to reduce micronutrient deficiencies?. Front. Plant Sci. 4:429. doi: 10.3389/fpls.2013.00429

This article was submitted to Plant Physiology, a section of the journal Frontiers in Plant Science.

Copyright (c) 2013 Murgia, De Gara and Grusak. This is an open-access article distributed under the terms of the Creative Commons Attribution License (CC BY). The use, distribution or reproduction in other forums is permitted, provided the original author(s) or licensor are credited and that the original publication in this journal is cited, in accordance with accepted academic practice. No use, distribution or reproduction is permitted which does not comply with these terms. 